\title{
Compreensão de construções passivas em crianças com perturbação do espectro do autismo $^{1}$
}

\author{
Raquel Costa \& Maria Lobo \\ Faculdade de Ciências Sociais e Humanas - Universidade Nova de Lisboa \\ Centro de Linguística da Universidade Nova de Lisboa
}

\begin{abstract}
:
This investigation aims to study the comprehension of passives in children with Autism Spectrum Disorder (ASD). Research on passives is sparse and remains inconclusive. Fourteen Portuguese speaking children with ASD (between ages 7 and 13), including children with low and high non-verbal IQ, were compared to four typically developing (TD) children. The comprehension of various types of passives (short vs long, eventive vs psychological) was assessed via a picture selection task. Most children with ASD showed difficulties with passive constructions as compared to controls and to previous studies with Portuguese TD children. The results are in line with the ones we find in studies with this population conducted in other languages. Furthermore, difficulties with passives were dissociated from nonverbal abilities.
\end{abstract}

Keywords: passives; autism; language acquisition; syntax

Palavras-chave: passivas, autismo, aquisição da linguagem, sintaxe

\section{Introdução}

Neste estudo investigamos a compreensão de construções passivas em crianças com perturbação do espectro do autismo (PEA). A investigação sobre as competências linguísticas destas crianças suscita interesse, uma vez que as PEA são a segunda mais frequente perturbação global de desenvolvimento (DSM-V, American Psychiatric Association, 2013; US EPA, 2019). Apesar da enorme variabilidade encontrada nas manifestações da patologia, a linguagem é uma das componentes que está frequentemente afetada.

Ao longo de vários anos, a investigação das competências linguísticas em crianças diagnosticadas com PEA centrou-se no estudo das competências pragmáticas, uma vez que, apesar da enorme diversidade das manifestações clínicas da patologia, a falta de interesse na interação social é uma característica comum a todas as crianças diagnosticadas com PEA (Klin, 2006). Recentemente, temos assistido a um maior interesse em investigar as competências sintáticas destas crianças. No entanto, os estudos realizados têm obtido informações contraditórias e são praticamente inexistentes em crianças falantes de português europeu (PE).

Os únicos estudos que conhecemos que se centram especificamente em estruturas sintáticas, nomeadamente relativas e estruturas de controlo, são os de Martins, Santos e Duarte (2017) e (2018), que retomamos na próxima secção.

Tanto quanto sabemos, não existem estudos para o PE que investiguem a produção e a compreensão de passivas em crianças com PEA. O grande objetivo desta investigação será assim contribuir para determinar se os défices encontrados em crianças com PEA falantes de PE vão ao encontro dos dados obtidos para crianças com a mesma patologia falantes de outras línguas com as mesmas características sintáticas. Estas descobertas serão importantes também para desenvolver formas de avaliação e intervenção terapêutica mais eficazes.

\footnotetext{
${ }^{1}$ Este trabalho foi realizado com o apoio da Fundação para a Ciência e Tecnologia, através de bolsa de doutoramento atribuída a Raquel Costa - PD/BD/128136/2016.
} 


\subsection{Perturbações do Espectro do Autismo - definição e características}

As PEA são definidas como uma síndrome neurocomportamental com origem em perturbações do sistema nervoso central que afeta o normal desenvolvimento da criança, caracterizada por dificuldades ao nível das interações sociais, da comunicação e presença de padrões de comportamento, interesses e atividades estereotipados, restritos e repetitivos. Os primeiros sintomas emergem geralmente entre os 6 e os 18 meses, mas, devido à marcada heterogeneidade da sua manifestação, o diagnóstico é difícil, muitas vezes tardio, sendo inclusive possível encontrar adultos a conviver com o autismo sem terem sido diagnosticados (ICD-10, World Health Organisation, 1992; DSM-V, American Psychiatric Association, 2013).

De acordo com o DSM-V, os sintomas incluem dois grandes domínios de perturbação: o domínio da interação social e comunicação e o domínio comportamental. Estas perturbações podem ser ou não acompanhadas de défice cognitivo, perturbação da linguagem e/ou outra alteração do neurodesenvolvimento, comportamental ou mental (American Psychiatric Association, 2013). Tendo por base as competências cognitivas, os indivíduos com autismo são classificados como de alto-funcionamento e de baixo-funcionamento.

Os estudos epidemiológicos realizados em vários países apresentam números muito díspares e ao longo dos anos parece observar-se um aumento da prevalência, acreditando-se que ambas as situações serão originadas por diferenças nos critérios de inclusão e por uma maior sensibilização para a patologia (Wing e Potter, 2002). Estima-se que a perturbação terá uma prevalência mais elevada nas crianças do sexo masculino, tendo Klin (2006) determinado um rácio de 3,5 a 5,1 rapazes por cada rapariga. Nos EUA, em 2014, uma em cada 59 crianças estava diagnosticada com PEA, com um rácio de 4 rapazes para uma rapariga, o que significa que 1 em cada 39 crianças do sexo masculino estava diagnosticada com a patologia (Baio et al., 2018). Em Portugal, foi realizado um estudo epidemiológico, em 2007, que apontou para uma prevalência de 9.2/10000 no continente e 15.6/10000 nos Açores (Oliveira et al., 2007). Fombonne (2009) realizou uma revisão sistemática da literatura, tendo determinado uma prevalência a nível mundial de 60 a 70/10000, o que significa que 1 em cada 152 crianças é afetada pela patologia.

Como já foi referido anteriormente, a manifestação da patologia é muito heterogénea, como tal as competências linguísticas observadas também partilham esta heterogeneidade e a sua caracterização não tem sido consensual entre os investigadores. Tager-Flusberg \& Joseph (2003) identificaram dois fenótipos da linguagem: crianças com competências linguísticas dentro dos parâmetros normais e crianças com alterações da linguagem semelhantes às observadas em crianças com perturbação específica da linguagem (PEL), mais recentemente designada como perturbação do desenvolvimento da linguagem (PDL) (Bishop, 2017). Estes autores, não esquecendo que existe um número significativo de crianças que nunca adquire oralidade, admitiam ainda que se podiam caracterizar outros subgrupos. Eigsti, Bennetto e Dadlani (2007), por sua vez, dividiram as crianças com PEA em 4 grupos: crianças com ausência de oralidade, crianças com atraso no desenvolvimento da linguagem, crianças com características desviantes na produção da linguagem (ecolália e jargão) e crianças com boas competências discursivas e pragmáticas. Mais recentemente, Wittke et al. (2017) estudaram o discurso espontâneo de 82 crianças com 5 anos de idade diagnosticadas com PEA falantes de inglês e/ou espanhol e definiram três subgrupos linguísticos: crianças com um desenvolvimento típico da linguagem, crianças com dificuldades na produção gramatical mas com vocabulário intacto e crianças com baixas competências gramaticais.

Contudo, nem todos os estudos são consensuais quanto à existência ou não de problemas sintáticos e quanto à natureza desses problemas em crianças com PEA.

Vários autores têm identificado um desenvolvimento atípico na aquisição da sintaxe com determinados défices gramaticais. Eigsti, Bennetto e Dadlani (2007), num estudo com crianças com PEA, falantes de inglês, encontraram omissão de palavras funcionais, dificuldades no uso dos morfemas de pessoa e tempo, atraso no desenvolvimento sintático em geral, assim como um atraso significativo quando comparadas com crianças da mesma idade cronológica e idade mental não-verbal. Num outro estudo, Eigsti \& Bennetto (2009) concluíram que as crianças com esta patologia apresentam uma menor sensibilidade à terceira pessoa do singular e ao 
marcador do presente progressivo. Estes resultados diferem, no entanto, dos encontrados em crianças com PEL/PDL, concluindo os autores que estaremos perante dois grupos diferentes quanto ao desenvolvimento da linguagem. Goodwin et al. (2012) estudaram a compreensão de interrogativas de sujeito e de objeto em crianças com PEA falantes de inglês e concluíram que estas estruturas se desenvolvem mais tardiamente em crianças com PEA do que em crianças com desenvolvimento típico (DT). Durrleman et al. (2015), na sua investigação com crianças falantes de francês, concluíram que as crianças com PEA sem atraso de desenvolvimento da linguagem apresentam uma prestação pior do que os controlos da mesma idade na compreensão de orações relativas de objeto e que as crianças com PEA com atraso de desenvolvimento da linguagem apresentam mais dificuldades nas orações relativas de sujeito do que as crianças com PEA sem atraso. Os investigadores também concluíram que as dificuldades gramaticais se mantêm até à idade adulta em adultos com PEA com QI normal. Os autores referem ainda que estes défices, quando ligeiros, não são na maioria das vezes detetados pelos testes estandardizados de avaliação da linguagem.

Há, contudo, autores que defendem que as competências gramaticais das crianças com PEA estão intactas (Kjelgaard e Tager-Flusberg, 2001; Tek et al., 2014; Whyte, Nelson \& Scherf, 2014). Whyte et al. (2014), por exemplo, concluíram que, desde que as crianças apresentem a mesma idade cognitiva, a sua performance em tarefas sintáticas é comparável à performance das crianças sem alterações.

Como já foi referido, a amostra das investigações acerca das competências sintáticas em crianças com PEA é constituída sobretudo por falantes do inglês. As investigações para a língua portuguesa são escassas. Para o português do Brasil, Barreira (2011) avaliou a morfologia e a sintaxe de indivíduos com autismo de alto funcionamento através de um teste de avaliação de linguagem estandardizado e concluiu que os sujeitos demonstraram mais dificuldade em produzir conjunções subordinadas e coordenadas e menos dificuldades com substantivos, verbos, adjetivos ou pronomes indefinidos.

Para o PE, Martins, Santos \& Duarte (2017), como foi referido anteriormente, estudaram a produção (através de uma tarefa de repetição de frases) e compreensão (através de uma tarefa de juízo de valor de verdade) de relativas de sujeito e de objeto com extração curta e extração longa em crianças com PEA, comparativamente a crianças com PEL/PDL e DT. Os resultados obtidos demonstram que as crianças apresentam, até a uma idade posterior à observada em crianças com DT, mais dificuldades em compreender relativas de objeto e que o grupo com PEA tem mais dificuldades com a extração longa. Martins, Santos \& Duarte (2018) investigaram a compreensão de orações relativas de sujeito e de objeto, assim como a compreensão de estruturas de controlo de sujeito e de objeto por crianças com PEA de alto funcionamento, comparando-as com crianças com PEL/PDL e com DT. As autoras mostram que não só as estruturas com movimento são problemáticas nas crianças com PEA: também alguns tipos de estruturas de controlo se revelaram difíceis.

\subsection{A passiva}

\subsubsection{A estrutura passiva}

A aquisição da construção passiva é um dos tópicos a que os linguistas têm dado grande atenção, sobretudo por não existir consenso em relação às causas que estarão na origem de uma aquisição tardia na maioria das línguas (Armon-Lotem et al., 2016).

As construções passivas podem ser de três tipos diferentes: passivas sintáticas (eventivas, verbais, perifrásticas, participiais), passivas pronominais (de -se ou reflexas) e passivas adjetivais, resultativas ou de estado (Mateus et al., 2003).

Nas passivas sintáticas, o constituinte com a função de sujeito na frase passiva tem, na ativa correspondente, a função de complemento direto e o constituinte introduzido pela preposição por na passiva (complemento agente da passiva) tem a função de sujeito na frase ativa correspondente. Existe constância de papel temático entre o sujeito da passiva e o complemento direto da ativa, assim como entre o complemento agente 
da passiva e o sujeito da ativa. O SN introduzido pela preposição por é opcional. Esta opcionalidade poderia levar a concluir que se trataria de um adjunto. No entanto, mesmo nas passivas em que este complemento não é foneticamente realizado (as chamadas passivas curtas (1)), o papel temático externo está implícito, como mostra a possibilidade de ocorrer um advérbio orientado para o agente como deliberadamente (2) ou um modificador que expressa uma finalidade (3).

(1) A bebé foi beijada (pela mãe).

(2) O cão foi deliberadamente atropelado.

(3) A janela foi aberta para arejar a sala.

Nas passivas adjetivais, tal como nas anteriores, o constituinte com a relação gramatical de sujeito corresponde ao argumento interno, complemento direto do verbo a partir do qual a forma participial é formada. As passivas adjetivais incluem os verbos estar e ficar associados a um particípio passado. No entanto, de acordo com Mateus et al. (2003), este tipo de construção apresenta características distintas das passivas sintáticas: a mudança de categoria do verbo para adjetivo; a eliminação do papel temático do argumento externo (não permite a ocorrência do constituinte a que se costuma chamar "agente da passiva"). Este tipo de passivas pode ser dividido em resultativas (4) e estativas (5).

(4) O exemplo ficou corrigido.

(5) O exemplo está correto.

Nas passivas pronominais, de -se ou reflexas, o argumento interno direto do verbo tem relação gramatical de sujeito, sendo ele que controla a concordância verbal, e tem obrigatoriamente traços de $3^{\mathrm{a}}$ pessoa gramatical. Nestas passivas existe constância de papéis temáticos entre o constituinte que ocorre como sujeito e o constituinte com o papel temático interno direto da ativa correspondente e, na análise de Mateus et al. (2003), é o clítico -se que recebe o papel temático externo do verbo. Em português, tal como nas restantes línguas com sujeito nulo, são mais frequentes as passivas de -se impessoais (6), com o argumento interno direto indefinido ou constituído por um nome simples (Mateus et al., 2003).

(6) Os artigos publicaram-se.

Esta investigação debruçar-se-á apenas sobre as passivas sintáticas/eventivas.

\subsubsection{Hipóteses explicativas da aquisição tardia da passiva}

Já em Bever (1970) se mostrava que as construções passivas reversíveis eram estruturas de aquisição tardia, possivelmente pela alteração da ordem canónica dos argumentos nas frases. Borer \& Wexler (1987) tentaram justificar a aquisição tardia da passiva através da ideia de que as cadeias argumentais (cadeias-A) ${ }^{2}$ são de desenvolvimento tardio, estando sujeitas a maturação - Hipótese da Maturação. Para esta justificação ser válida seria necessário que a aquisição tardia das cadeias-A fosse universal, não sendo possível uma criança adquirir uma estrutura que exija cadeias-A e não adquirir uma outra. Contudo, Costa \& Friedmann (2012) mostram que crianças com menos de dois anos analisam adequadamente as construções com inacusativos e conseguem mover o argumento dos inacusativos da posição de objeto para a de sujeito, formando, portanto, cadeias-A. Estes

\footnotetext{
${ }^{2}$ Uma cadeia-A é formada quando existe movimento de uma posição argumental para outra posição argumental. O movimento de um argumento interno para a posição de sujeito numa construção passiva ou numa estrutura inacusativa dá origem a uma cadeia-A.
} 
resultados indicam que este tipo de movimento é adquirido precocemente, o que põe em causa a hipótese defendida por Borer \& Wexler.

Babyonyshev et al. (2001) e Babyonyshev \& Brun (2004) reformularam esta teoria através da Hipótese de Requisito de Argumento Externo (External Argument Requirement Hypothesis), teoria que defende que, em sistemas imaturos, são agramaticais as estruturas que carecem de argumento externo. Assim, as crianças teriam dificuldade com passivas eventivas, mas não com passivas adjetivais, que não requerem a formação de uma cadeia-A.

Uma explicação diferente é dada por Fox \& Grodzinsky (1998). Os autores observam que existem dificuldades apenas na compreensão de passivas com verbos não eventivos e com complemento agente da passiva (sintagma-por) expresso. Os autores propõem a hipótese de que as crianças não têm dificuldade com cadeiasA, mas antes com a transmissão do papel temático do argumento externo ao sintagma-por. Só no caso dos verbos eventivos é que a preposição por (no inglês, by) será capaz de atribuir papel temático relevante..

Mais tarde, Wexler (2004) e Hirsch \& Wexler (2006), já num enquadramento minimalista (Chomsky, 1995), reformularam a Hipótese de Défice de Cadeias-A, defendendo que o atraso na aquisição da passiva não está relacionado com a formação de cadeias-A, mas sim com o facto de as crianças assumirem que todas as categorias vP e CP são fases fortes, o que torna as passivas, inacusativos e estruturas de elevação agramaticais. Esta restrição (Universal Phase Requirement) estaria ativa na gramática das crianças até pelo menos aos 5 anos e amadureceria entre os 6 e os 8 anos. De acordo com Chomsky (2001), o movimento de um constituinte para fora de uma fase (vP ou CP), só é permitido se o constituinte tiver sido primeiro movido para a periferia esquerda da fase, uma vez que apenas o limite (núcleo e especificadores) permanece acessível a operações que são requeridas na fase seguinte. Uma vez que as crianças assumem o v, quer seja ou não defetivo, como sendo uma fase forte, o argumento interno está inacessível à fase superior, não sendo possível realizar as operações de Agree nem Move para T. A derivação falha porque T mantém-se com elementos que não são verificados, tornando as passivas agramaticais para as crianças. Estas estruturas de elevação são aceites na gramática do adulto, porque o vP relevante não define fases fortes (é fraco, defetivo).

Por sua vez, Hyams \& Snyder (2005), inspirando-se na análise de Collins (2005), defendem que as dificuldades com a passiva são atribuíveis a efeitos de intervenção. Se o argumento externo da passiva é projetado sintaticamente, o movimento do objeto cruza a posição do argumento externo interveniente, o que constitui uma violação do Princípio da Minimalidade Relativizada. Collins (2005) propõe, por isso, que a intervenção aqui é evitada através de uma operação adicional, que implica um movimento preliminar à esquerda de um VP chunk contendo o verbo e o objeto e excluindo o argumento externo. Deu a este processo o nome de smuggling. Na hipótese de Hyams \& Snyder (2005), este processo parece necessitar de algum tempo para se desenvolver e as crianças, em estádios iniciais, não terão acesso a este processo, que se tornará disponível por volta dos 4 anos de idade. Os autores apresentam a Universal Freezing Hypothesis, defendendo que as crianças sobregeneralizam o Freezing Principle a casos de movimento que implicam smuggling sobre um experienciador, como é o caso das passivas, ou seja, não lhe admitem nenhuma exceção, sendo que o smuggling é uma exceção a este princípio.

Independentemente da análise teórica adotada, os trabalhos têm mostrado dificuldades na compreensão de passivas que são mais acentuadas com passivas longas e com passivas não eventivas. Num estudo interlinguístico, que procurou estudar a aquisição da passiva em 11 línguas, concluiu-se que as passivas curtas são compreendidas mais facilmente do que as passivas longas, levantando a hipótese de que as crianças interpretam as passivas curtas como adjetivais, sendo que as passivas adjetivais são compreendidas mais cedo do que as sintáticas. Na tarefa que avalia as passivas longas, são apresentadas imagens com três personagens e apresenta-se uma frase em que uma personagem sofre uma ação por parte da outra personagem. Se a criança não dominar a construção passiva, tem duas possibilidades para a interpretar: ou ignora a preposição e interpreta a frase como ativa, realizando a inversão de papéis temáticos, ou considera que a preposição introduz um locativo e não um 
argumento externo e pode escolher a terceira personagem. Nos casos em que as crianças escolheram a resposta errada, optaram maioritariamente pela imagem que ilustrava a situação inversa, revelando uma preferência pela ordem canónica das frases (Armon-Lotem et al., 2016).

Outros autores têm considerado o papel das metodologias usadas nos piores resultados obtidos com passivas não eventivas e também o papel de capacidades de processamento mais gerais. Volpato, Verin \& Cardinaletti (2016) sugerem que as dificuldades com as passivas não agentivas estarão sobretudo relacionadas com as dificuldades em representar através de imagens estes verbos. Da mesma forma, defendem que as dificuldades observadas com as passivas longas em crianças mais novas estarão sobretudo relacionadas com limitações ao nível da memória de trabalho.

\subsubsection{Dados sobre a compreensão da passiva em crianças com DT falantes de português}

Para o português, há já alguns estudos sobre a aquisição de passivas em crianças com DT, falantes ou da variedade brasileira ou da variedade europeia.

Rubin (2004), num estudo realizado para o português do Brasil com crianças entre os 5,7 e os 6,6 anos de idade, concluiu que as crianças nestas idades já compreendem a passiva reversível e não reversível com verbos de ação, mas não compreendem a passiva com verbos não agentivos e apresentam muita dificuldade em distinguir as passivas longas (reversíveis e não reversíveis) das ativas.

Lima Júnior (2012), por sua vez, levanta a possibilidade de a diferença entre verbos agentivos e não agentivos se esbater frente a verbos psicológicos que impliquem uma leitura resultativa, ou seja, que permitam um traço de afetação com mudança de estado do SN (verbos como assustar, magoar, irritar, animar), verbos que permitem o mesmo desempenho do que o alcançado com verbos agentivos. Por sua vez, os verbos que não permitem um traço de afetação, tais como ver, ouvir, admirar e respeitar, revelam-se mais difíceis. O autor defende ainda que as crianças nem sempre recorrem à estratégia de atribuir papel temático de agente ao primeiro SN. Corrêa et al. (2017) também defendem que as dificuldades na atribuição do papel temático surgem apenas nas passivas longas.

Numa investigação levada a cabo por Estrela (2013), concluiu-se que as crianças falantes de PE compreendem as passivas aos 4 anos, sendo indiferente tratar-se de passivas curtas ou longas. $\mathrm{O}$ que interfere na compreensão é a presença de um verbo agentivo ou não agentivo. A autora no seu estudo concluiu que com 3 anos as crianças apresentam uma taxa de acerto de $63 \%$, com 4 anos de $77 \%$ e com 5 anos de $91 \%$. Ao considerar o tipo de verbo, verificou que com verbos agentivos as crianças de 3 anos apresentaram uma percentagem de acerto de $50 \%$, as de 4 anos de $79 \%$ e as de 5 anos de $93 \%$. Nas passivas com verbos não agentivos as percentagens de acerto foram mais baixas aos 4 e 5 anos, tendo as crianças de 3 anos apresentado uma percentagem de acerto de $50 \%$, as de 4 anos de $50 \%$ e as de 5 anos de $64 \%$. Mais recentemente, Agostinho (2020) na sua tese de doutoramento, apurou percentagens de acerto de $94,1 \%$ nas passivas curtas agentivas e de $62,5 \%$ nas longas, subindo esta percentagem para $88,4 \%$ aos 4 anos e ultrapassando os $90 \%$ aos 5 anos. Nas passivas curtas não agentivas, a percentagem de acerto foi de $42,1 \%$ aos 3 anos de idade, subindo para os $63,4 \%$ aos 4 anos e chegando aos 70,2\% aos 5 anos. Nas passivas longas não agentivas, a percentagem de acerto aos 3 anos de idade foi de $34,2 \%$, perto dos $50 \%$ aos 4 e 5 anos, ultrapassando apenas os $70 \%$ aos 7 anos (73,7\%). Confirma, por conseguinte, na generalidade, os resultados obtidos por Estrela (2013): maior dificuldade com passivas não agentivas comparativamente a passivas agentivas, sendo que as crianças compreendem as passivas agentivas, curtas e longas, aos 4 anos de idade, verificando-se um atraso na compreensão de passivas não agentivas.

\subsubsection{Dados da compreensão da passiva - PEA}


A compreensão da passiva em crianças com PEA também tem sido alvo de investigação. Alguns estudos (Tager-Flusberg, 1981; Paul, Fischer \& Cohen, 1988) sugerem que as crianças com PEA utilizam essencialmente a estratégia da ordem das palavras (a ordem nome-verbo-nome corresponde a agente-ação-objeto) para descodificar a passiva, o que conduz a uma má interpretação desta estrutura. Os autores referem que estas crianças apresentam dificuldade em utilizar a estratégia probable event, que consiste em atribuir o papel de agente a nomes animados e de objeto a nomes inanimados, o que poderá indicar que estas crianças têm boas competências sintáticas, mas dificuldade em utilizar a informação semântica. Estudos para a língua inglesa sugerem uma grande discrepância na idade de aquisição das passivas por crianças com e sem patologia. Estas últimas adquirem a estrutura muito mais cedo. Entre as crianças com PEA há também uma grande diferença consoante o nível cognitivo das crianças: crianças com QI abaixo da média apresentam mais dificuldade, tendo conseguido uma taxa de acerto nas passivas curtas e longas com verbos agentivos de $36 \%$ e de $30 \%$ nas passivas curtas com verbos não agentivos e de $26 \%$ nas longas. As crianças diagnosticadas com Síndrome de Asperger, crianças com QI normal a elevado, apresentaram as seguintes taxas de acerto: 83\% nas passivas, longas e curtas, com verbos agentivos, $100 \%$ nas passivas curtas não agentivas e 67\% nas passivas longas não agentivas (Perovic, Modyanova, Wexler, 2007).

Já Schroeder (2013) concluiu que as crianças dinamarquesas com PEA de alto funcionamento não apresentam dificuldade na compreensão das passivas, quer curtas quer longas (uma taxa de acerto de 91 a 100\%). Na realidade, apresentaram inclusivamente uma melhor prestação do que as crianças com DT. É, no entanto, necessário ressalvar que a amostra desta investigação, além de extremamente reduzida, era constituída por crianças em média mais velhas do que as de DT (7 participantes com idades entre os 13 e os 18 anos com QI acima da média). Este estudo indica também que estas crianças apresentam mais dificuldade nas passivas longas do que nas curtas.

Da mesma forma, Gavarró e Heshmati (2014) concluíram, na sua investigação, que as crianças falantes de persa com PEA de baixo-funcionamento apresentam uma performance significativamente pior (40\% nas passivas curtas e $51 \%$ nas passivas longas) do que as crianças com DT ( $98 \%$ curtas e $91 \%$ longas) e do que as crianças diagnosticadas com PEA de alto funcionamento (91\% curtas e $76 \%$ longas).

Os dados obtidos para o grego por Terzi et al. (2014) revelam que a aquisição é igualmente tardia em ambos os grupos: quer em crianças com PEA, que apresentaram uma taxa de acerto de 66,6\%, quer em crianças com DT, que obtiveram uma taxa de acerto de $70 \%$.

Por sua vez, Durrleman et al. (2017) definiram dois grupos distintos num grupo de crianças falantes de francês: um constituído por crianças com desenvolvimento da linguagem dentro dos parâmetros normais, que tiveram uma performance semelhante à das crianças com DT, e outro grupo constituído por crianças com perturbação da linguagem, que apresentaram uma performance significativamente pior. As crianças com PEA falantes da língua francesa apresentaram melhor performance com verbos agentivos $(60 \%)$ do que não agentivos (21\%), não se tendo observado diferenças significativas entre passivas curtas (62\%) e longas (56\%).

Em jeito de síntese, podemos afirmar que as investigações realizadas até ao momento nas várias línguas têm chegado a resultados muito díspares. Assim, há estudos que defendem que as crianças com DT adquirem a estrutura passiva mais cedo do que as crianças com PEA (Perovic, Modyanova \& Wexler, 2013; Gavarró \& Heshmati, 2014), enquanto há autores que defendem que, se as crianças com PEA tiverem um QI elevado, os resultados obtidos não diferem dos observados em crianças com DT (Schroeder, 2013). No que diz respeito às crianças com PEA há autores que distinguem dois grupos com base nas competências cognitivas: crianças com QI baixo, que apresentam graves dificuldades na compreensão de passivas, e crianças com PEA de QI elevado, cujas dificuldades são menos marcadas (Perovic, Modyanova e Wexler, 2013; Gavarró e Heshmati, 2014). Por outro lado, há quem considere que as competências cognitivas não têm influência nos resultados, defendendo que o que interfere nos resultados são as competências linguísticas (Durrleman et al., 2016). Estes autores também dividem as crianças em dois grupos, mas desta feita com base nas competências linguísticas: um grupo 
com alterações ao nível da linguagem, que apresenta dificuldade na compreensão da estrutura passiva; outro grupo sem alterações da linguagem, que apresenta uma performance semelhante às crianças com DT. Há ainda quem defenda que a compreensão da estrutura passiva é igualmente tardia em crianças com DT e PEA (Terzi et al., 2014).

Da mesma forma, e de forma semelhante ao que se verifica nos dados obtidos em estudos apenas com crianças com DT, verificamos que tanto há autores que defendem que as passivas curtas são mais acessíveis do que as passivas longas, como há autores que defendem que não há diferenças significativas entre este tipo de passivas. Parece ser consensual que o que interfere na compreensão é o tipo de verbo, sendo os verbos agentivos mais acessíveis do que os não agentivos.

Nas próximas secções, procuraremos contribuir para o conhecimento da aquisição da passiva em crianças com PEA num estudo com crianças falantes do PE, língua em que passivas eventivas se distinguem das passivas adjetivais através do verbo auxiliar.

\section{2. $O$ estudo}

\subsection{Questões de investigação}

Os objetivos desta investigação são: i) determinar se as PEA estarão associadas a problemas de desenvolvimento sintático e, neste estudo em particular, na compreensão de passivas; ii) averiguar se existem perfis diferentes de crianças com PEA; iii) verificar se existe uma correlação entre o desenvolvimento cognitivo e o desenvolvimento linguístico.

Tendo em conta os estudos já realizados, quer com crianças com DT quer com crianças diagnosticadas com PEA, tentaremos confirmar as seguintes hipóteses:

Hipótese 1: As crianças com PEA com QI baixo apresentam mais dificuldades na compreensão da estrutura passiva do que as crianças com DT.

Hipótese 2: As crianças com PEA com QI médio a elevado apresentam uma performance na compreensão da estrutura passiva semelhante à encontrada em crianças com DT com a mesma idade cronológica.

Hipótese 3: As crianças com PEA de QI médio a elevado não apresentam diferenças na compreensão de passivas longas e curtas.

Hipótese 4: As crianças com PEA com QI médio a elevado apresentam maiores dificuldades na compreensão de passivas com verbos não agentivos do que com verbos agentivos.

\subsection{Metodologia}

Com o objetivo de responder a estas questões, foi aplicada uma tarefa de seleção de imagens elaborada para o efeito. As crianças realizaram a tarefa sem limitação de tempo. A recolha de dados foi interrompida sempre que a criança demonstrou sinais de cansaço ou distração.

Para avaliar a compreensão de passivas foi adaptado um teste de seleção de imagens de Moita (no prelo). $\mathrm{O}$ teste apresenta um design $2 \times 2$ e procura avaliar as condições passivas longas e passivas curtas. Cada uma destas condições apresenta dois níveis: verbos agentivos e verbos não agentivos. Assim, o teste é composto por 40 itens experimentais:

a) 10 passivas curtas agentivas; 
Exemplo: "O pai é coçado."

b) 10 passivas curtas não agentivas; Exemplo: "O pai é amado."

c) 10 passivas longas agentivas;

Exemplo: "A mãe é penteada pela avó."

d) 10 passivas longas não agentivas

Exemplo: "O pai é ouvido pela avó."

Os verbos agentivos selecionados foram: pentear, coçar, abraçar, beijar e empurrar; os verbos não agentivos foram: odiar, amar, adorar, ver e ouvir. ${ }^{3}$

Nas passivas longas (Figura 1), numa das imagens pode ser observado o agente a realizar a ação; noutra o paciente a realizar a ação (inversão do papel temático); e na terceira outra personagem a praticar a ação.
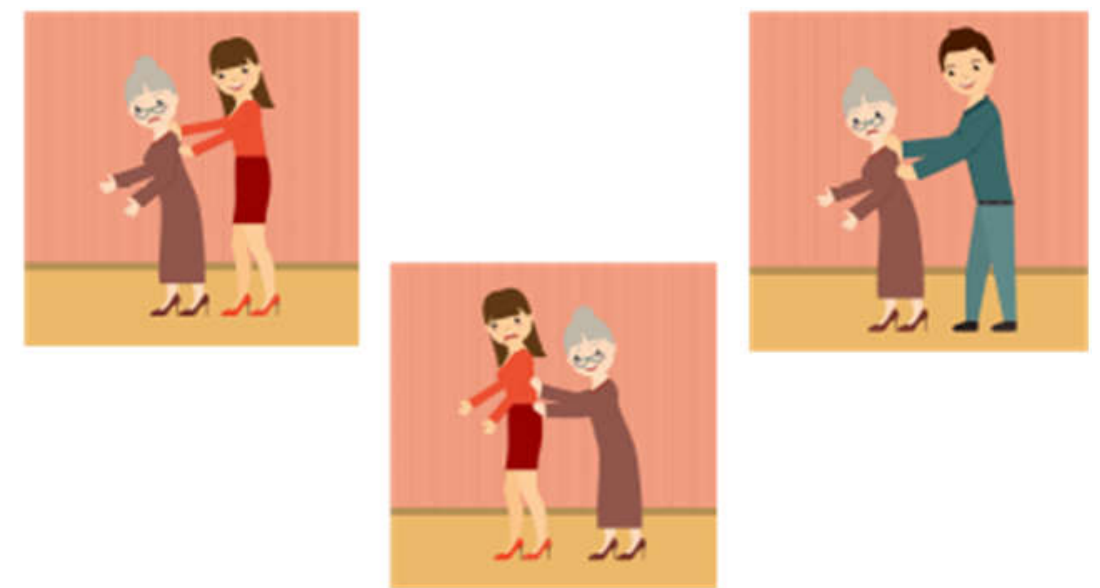

Figura 1. Exemplo de item de teste para passiva longa "A avó é empurrada pela mãe."

Nas passivas curtas (Figura 2), a criança tem de escolher também entre três imagens, mas neste caso as imagens apresentadas são uma imagem-alvo, com o agente a praticar a ação, e duas imagens em que surge o paciente a praticar a ação.

\footnotetext{
${ }^{3}$ Nos estímulos, usou-se o presente do indicativo. Admite-se que a utilização do presente do indicativo nas construções passivas seja menos natural do que a utilização progressivo. No entanto, os resultados observados nas crianças com DT testadas até ao momento, assim como a utilização do presente do indicativo nas frases de controlo na ativa, adicionadas à versão revista da tarefa, parecem indicar que não é o tempo verbal que estará na origem das dificuldades.
} 

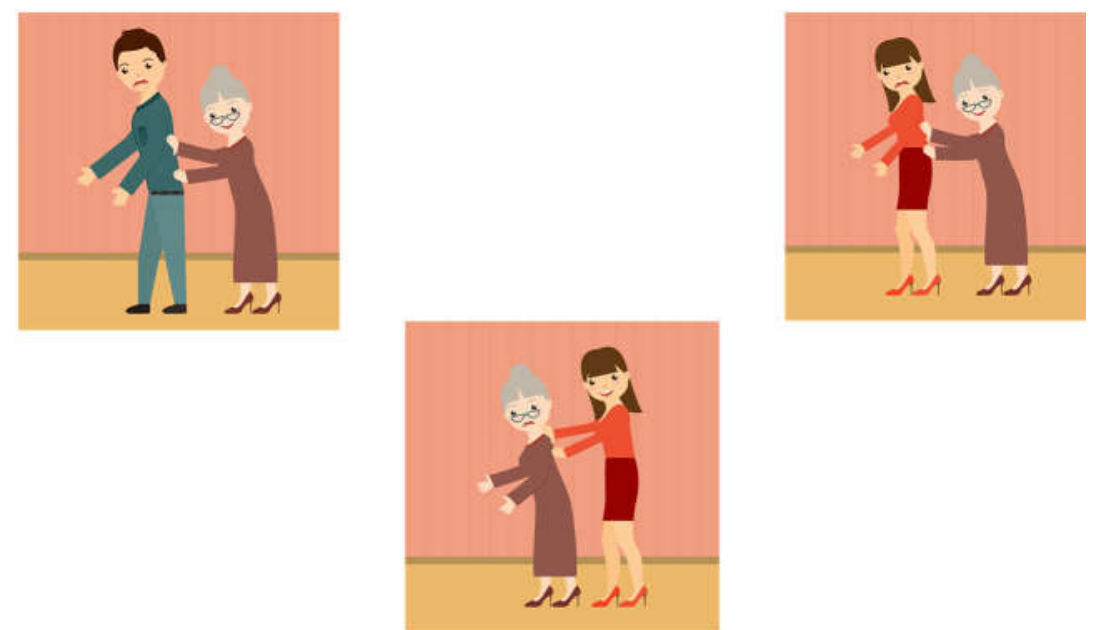

Figura 2. Exemplo de item de teste para passiva curta "A avó é empurrada."

Antes de se iniciar o teste, foram apresentadas as personagens - uma família com uma mãe, um pai, uma avó, um bebé e um cão - e a criança foi convidada a jogar um jogo: "Vamos jogar novamente 4 o jogo da família. Neste jogo não tens de dizer nada. Eu vou mostrar três imagens e dizer uma frase e tu vais apontar para a imagem da qual eu estou a falar." Inicialmente, foi realizado um item de treino para garantir que a criança tinha compreendido a tarefa.

Este estudo insere-se num projeto de doutoramento em que também estão a ser recolhidos dados da produção e da compreensão de outras estruturas sintáticas, nomeadamente dos pronomes clíticos, interrogativas-Q e relativas. Como forma de motivação, antes de iniciarem as tarefas de recolha de dados, as crianças mais novas foram convidadas a escolher um puzzle entre três temas à escolha (princesas, animais e carros) e receberam um postal com dois lados: um com 8 quadrados em branco para colarem as peças do puzzle, outro com uma imagem e a mensagem de agradecimento "Muito Obrigada!". Sempre que completavam uma tarefa, era-lhes oferecido uma parte/autocolante do puzzle.

As respostas foram codificadas em: 1 ("resposta correta"), 2 (resposta incorreta - inversão do papel temático") e 3 ("resposta incorreta - outra"). Nas passivas longas, a classificação "2" foi utilizada quando a criança selecionou a imagem com o paciente no lugar de agente e "3" quando escolheu a imagem em que surge outra personagem a praticar a ação. Nas passivas curtas, apenas foram utilizadas as classificações "1" para resposta correta e "3" para incorreta, uma vez que a situação inversão do papel temático não se aplica nesta condição.

Foram avaliadas até ao momento 14 crianças (2 crianças do sexo feminino e 12 do sexo masculino) diagnosticadas com PEA, monolingues, falantes de PE, com idades compreendidas entre os 7 anos e 2 meses e os 13 anos. Foram incluídas crianças com QI não verbal inferior a superior (todas as crianças foram avaliadas cognitivamente através do Teste de Matrizes Coloridas de Raven).

As crianças foram recrutadas através das Unidades de Ensino Estruturado para a Educação de Alunos com Perturbações do Espectro do Autismo, Associações de Apoio às PEA, instituições particulares de solidariedade social e clínicas privadas de avaliação e intervenção terapêutica.

\footnotetext{
${ }^{4}$ Antes de realizar a tarefa de compreensão de estruturas passivas, as crianças realizaram a tarefa de produção, daí a utilização do termo "novamente".
} 


\begin{tabular}{ccccc}
\hline & & QI (n) & Total \\
\cline { 2 - 5 } Idade (anos) & Inferior & Médio & Superior & 3 \\
\hline $\mathbf{7 - 8}$ & 1 & 1 & 1 & 4 \\
\hline $\mathbf{8 - 9}$ & 2 & 2 & - & 1 \\
\hline $\mathbf{9 - 1 0}$ & 0 & - & - & 2 \\
\hline $\mathbf{1 0 - 1 1}$ & 2 & - & - & 1 \\
\hline $\mathbf{1 1 - 1 2}$ & 1 & - & 1 & 3 \\
\hline $\mathbf{1 2 - 1 3}$ & 2 & $\mathbf{2}$ & $\mathbf{1 4}$ \\
\hline
\end{tabular}

Tabela 1. Número de crianças diagnosticadas com PEA por faixa etária e nível de QI não verbal

Testaram-se também 4 crianças monolingues falantes de PE, sem alterações auditivas, neurológicas, cognitivas ou de linguagem, entre os 6 anos e 10 meses e os 10 anos e os 2 meses, para verificar se estas estruturas estão adquiridas nas crianças com DT destas faixas etárias. ${ }^{5}$

\subsection{Resultados}

Uma vez que a amostra é bastante reduzida, os resultados obtidos até ao momento foram analisados sobretudo em termos de percentagens de acerto. Foi dada especial atenção à análise das prestações a nível individual, uma vez que o número da amostra por variável (faixa etária, sexo, QI não verbal) não permite outro tipo de análise estatística válida.

Como se pode observar na Tabela 2, as crianças diagnosticadas com PEA apresentaram uma taxa de acerto de $55,7 \%$ nas passivas longas e de $47,9 \%$ nas passivas curtas, percentagem bastante inferior à encontrada no grupo de controlo: 97,5\% passivas longas e de 100\% nas passivas curtas. Se olharmos para o tipo de verbo (estruturas agentivas vs. não agentivas), obtemos uma percentagem de 59,9\% nas passivas agentivas e de $45,1 \%$ nas passivas não agentivas, uma taxa também bastante inferior à observada no grupo de controlo: $98,75 \%$ nas passivas agentivas e 96,25 nas não agentivas.

\begin{tabular}{ccccc}
\hline $\begin{array}{c}\text { Tipo de Passiva/ } \\
\text { Grupo }\end{array}$ & Longas & Curtas & Agentivas & Não agentivas \\
\hline Controlo & 97,5 & 100 & 98,75 & 96,25 \\
\hline PEA & 55,7 & 47,9 & 59,9 & 45,1 \\
\hline
\end{tabular}

Tabela 2. Percentagem global de acerto por tipo de passiva

Se olharmos para a combinação das duas condições (Tabela 3), verificamos que no grupo de controlo os participantes obtiveram taxas de acerto de $100 \%$ nas passivas curtas, independentemente do tipo de verbo, situação que não se verificou com as passivas longas (é necessário ressalvar que apenas uma criança forneceu respostas incorretas, situação que poderá ter na sua origem distração). Verificamos também que a taxa de acerto na condição curtas não agentivas, no grupo das PEA, é bastante inferior a todas as outras.

\footnotetext{
${ }^{5}$ Este estudo encontra-se ainda em curso. Pretende-se alargar, de futuro, o número de crianças do grupo de controlo. Apesar de o número de crianças com DT testadas ainda ser reduzido, já permite verificar uma discrepância entre as crianças com DT e as crianças com PEA.
} 


\begin{tabular}{ccccc}
\hline $\begin{array}{c}\text { Tipo de Passiva/ } \\
\text { Grupo }\end{array}$ & $\begin{array}{c}\text { Longas } \\
\text { Agentivas }\end{array}$ & $\begin{array}{c}\text { Curtas } \\
\text { Agentivas }\end{array}$ & $\begin{array}{c}\text { Longas Não } \\
\text { agentivas }\end{array}$ & $\begin{array}{c}\text { Curtas } \\
\text { Não agentivas }\end{array}$ \\
\hline Controlo & 97,5 & 100 & 92,5 & 100 \\
\hline PEA & 61,1 & 58,8 & 51,3 & 39 \\
\hline
\end{tabular}

Tabela 3. Percentagem global de acerto por tipo de passiva.

\begin{tabular}{ccccc}
\hline QI & $\begin{array}{c}\text { Longas } \\
\text { agentivas }\end{array}$ & $\begin{array}{c}\text { Curtas } \\
\text { agentivas }\end{array}$ & $\begin{array}{c}\text { Longas } \\
\text { não agentivas }\end{array}$ & $\begin{array}{c}\text { Curtas } \\
\text { não agentivas }\end{array}$ \\
\hline$<\mathrm{P} 5(\mathrm{n}=8)$ & 55,6 & 63,9 & 44,4 & 38,7 \\
\hline $\mathrm{P} 25-50(\mathrm{n}=4)$ & 75 & 55,6 & 52,3 & 29,6 \\
\hline $\mathrm{P} 75(\mathrm{n}=2)$ & 55,6 & 50 & 77,3 & 54,6 \\
\hline
\end{tabular}

Analisando o grupo de crianças com PEA tendo por base as competências cognitivas, verificamos que o grupo de crianças com QI não verbal inferior obteve, ainda que as percentagens não sejam muito afastadas, melhores resultados na compreensão de passivas curtas agentivas e curtas não agentivas do que o grupo de QI não verbal médio, o que parece indicar que o QI não verbal não está diretamente relacionado com a prestação.

Se olharmos para os resultados individualmente, verificamos que as taxas de acerto variam entre os $0 \% \mathrm{e}$ os $100 \%$, o que atesta a heterogeneidade do grupo

Ao analisarmos as respostas nas passivas longas (o tipo de passiva em que é possível fazer esta análise), verificamos que a maioria das respostas erradas correspondeu à inversão do papel temático. Houve apenas sete respostas em que foi escolhida a imagem distratora, sendo que quatro das respostas foram dadas pelo mesmo participante, o que parece significar que as crianças apresentaram um bom nível de atenção.

Os participantes com as taxas de acerto mais elevadas não foram os participantes mais velhos: além do único participante que obteve percentagens de $100 \%$ em todas as condições, apenas dois participantes, com 7;7 e 8;0, obtiveram taxas de acerto de $100 \%$ nas passivas longas agentivas; o único participante que obteve uma percentagem de acerto superior a $70 \%$ nas passivas longas não agentivas tem $8 ; 11$, não sendo nenhum dos participantes referidos anteriormente; o participante que respondeu corretamente a todos os itens que avaliam as passivas curtas agentivas tem $10 ; 7$ e os três participantes que obtiveram taxas de acerto superiores a $80 \%$ têm $10 ; 0,7 ; 7$ e 8;0; nas passivas curtas não agentivas apenas três participantes apresentaram taxas de acerto superiores a $50 \%$, com idades de 10;0,7;7 e 11;5. Estes resultados parecem indicar que o fator idade não influi diretamente nos resultados.

Olhando para a dicotomia longas vs. curtas, verificamos que três participantes apresentam as mesmas percentagens de acerto nas duas condições e apenas três participantes apresentam uma maior percentagem de acerto em passivas curtas do que longas (no caso de um destes participantes as percentagens são muito próximas $50 \%-55 \%)$.

\section{Discussão}

Ao contrário das conclusões obtidas noutras investigações, que defendem que apenas as crianças com PEA com baixo QI têm problemas sintáticos (Perovic, Modyanova \& Wexler, 2007; Gavarró e Heshmati, 2014), os resultados obtidos até ao momento sugerem que as crianças diagnosticadas com PEA apresentam mais dificuldades do que o grupo de controlo na compreensão de todos os tipos de passivas. Estes resultados aproximam-se dos encontrados para crianças falantes de grego por Terzi et al. (2014). Apesar do número reduzido de participantes, o grupo de controlo parece não apresentar dificuldades na compreensão desta 
estrutura, resultados que vão ao encontro do esperado para as crianças falantes de PE com DT, que aos 5 anos e aos 7 anos já apresentam uma boa compreensão de passivas (Estrela, 2013; Agostinho, 2020), sendo que as crianças que participaram na investigação de Agostinho (2020) apenas aos 7 anos apresentaram taxas de acerto superiores a 70\% nas passivas longas com verbos não agentivos.

Não se confirma, desta forma, na totalidade, a hipótese 1, que previa que as crianças com PEA com QI baixo apresentariam maiores dificuldades na compreensão da estrutura passiva do que as crianças com DT. Esta hipótese não se confirma na totalidade, uma vez que tem implícita a ideia de que apenas as crianças com QI não verbal baixo teriam esta dificuldade, a qual não se verificaria em crianças com QI não verbal médio a elevado. Nesta investigação, ao contrário do observado noutros estudos (Perovic, Modyanova \& Wexler, 2007), o QI não verbal não se relacionou diretamente com a performance. Esta situação vai ao encontro do defendido por Durrleman et al. (2017) com base em dados de crianças falantes da língua francesa. Também neste estudo se mostrou não haver necessariamente relação entre o QI não verbal e o desempenho sintático das crianças com PEA.

Não se parece confirmar também a hipótese 2, que previa que as crianças com PEA com QI médio a elevado apresentariam uma performance na compreensão da estrutura passiva semelhante à encontrada em crianças com DT com a mesma idade cronológica. Os dados obtidos até ao momento revelam que as crianças com PEA manifestam, de uma forma geral, maiores dificuldades na compreensão da estrutura passiva do que o grupo de controlo.

Relativamente à hipótese 3, que previa que as crianças com PEA de QI médio a elevado não apresentariam diferenças na compreensão de passivas longas e curtas, podemos afirmar que, ainda que no caso das passivas com verbos agentivos as percentagens sejam aproximadas, parece existir uma tendência para uma maior dificuldade na interpretação de passivas curtas do que longas, ao contrário do verificado em crianças falantes de PE com DT e na investigação para a língua francesa, em que não se verificaram diferenças estatisticamente significativas entre estes tipos de passivas (Estrela, 2013; Durrleman et al., 2017). Curiosamente, as autoras que investigaram a aquisição da passiva em crianças falantes de persa também concluíram que as crianças com PEA e QI baixo apresentam maiores dificuldades em interpretar passivas curtas do que longas, situação que não se verificou com as crianças com DT e com as crianças com PEA de QI elevado (Gavarró e Heshmati, 2014). No entanto, a amostra reduzida da nossa investigação não permite determinar se a diferença referida é ou não estatisticamente significativa.

Respondendo à hipótese 4, que previa que as crianças com PEA apresentassem maiores dificuldades na compreensão de passivas com verbos não agentivos do que com verbos agentivos, podemos avançar que os dados obtidos até ao momento vão ao encontro dos resultados obtidos por Estrela (2013) e Agostinho (2020) para as crianças com DT, assim como das investigações com crianças com PEA falantes da língua inglesa (Perovic, Modyanova \& Wexler, 2007) e francesa (Durrleman et al., 2017): as passivas não agentivas são mais difíceis do que as agentivas.

\section{Conclusão}

Neste estudo, mostramos que as crianças com PEA parecem ter dificuldades em compreender a passiva. Apesar da amostra reduzida e consequentemente da impossibilidade de tirar conclusões robustas, os dados obtidos até ao momento parecem demonstrar uma forte tendência para que as competências sintáticas das crianças com PEA sejam independentes do QI não verbal.

Os dados sugerem que as passivas com verbos não agentivos são mais difíceis do que as passivas com verbos agentivos e parece haver uma possibilidade de as passivas curtas serem mais difíceis de interpretar do que as passivas longas. O tipo de erro mais frequente é a escolha da situação inversa (passivas longas).

É, no entanto, necessário ressalvar que a amostra desta investigação é bastante reduzida, sobretudo porque 
abrange várias faixas etárias e um grupo que se revelou muito heterogéneo, o que não permite tirar conclusões robustas, mas apenas sugerir uma tendência. Da mesma forma, o grupo de controlo é bastante reduzido e sem emparelhamento com o grupo de alvo quanto à idade e ao QI não verbal, o que se espera fazer no futuro.

Referimos ainda que não foi possível avaliar as competências linguísticas destas crianças através de instrumentos globais de avaliação da linguagem, o que se tem revelado essencial, noutras investigações, para definir os perfis de crianças diagnosticadas com PEA. Da mesma forma, é necessário referir que o nível de atenção poderá ter um papel decisivo nos resultados, mas devido à ausência de instrumentos que permitam medir o nível de atenção continuamente ao longo das tarefas, não é possível avaliar a interferência deste fator nos resultados. Este é, por conseguinte, um estudo preliminar, que precisará de ser desenvolvido em trabalho futuro.

\section{Referências:}

Agostinho, Celina (2020) The Acquisition of the passive in European Portuguese. Dissertação de Doutoramento, Universitat Autònoma de Barcelona.

American Psychiatric Association (2013) Diagnostic and statistical manual of mental disorders ( $5^{\mathrm{a}}$ edição) doi: 10.1176/appi.books.9780890425596

Armon-Lotem, Sharon et al. (2016) A large-scale cross-linguistic investigation of the acquisition of passive. Language Acquisition. 23(1), pp. 27-56. doi: 10.1080/10489223.2015.1047095

Babyonyshev, Maria, Ganger, Jennifer, Pesetsky, David, Wexler, Kenneth (2001) The Maturation of Grammatical Principles: Evidence from Russian Unaccusatives. Linguistic Inquiry. 32(1), pp. 1-44.

Babyonyshev, Maria, Brun, Dina (2004) The acquisition of perfective and imperfective passive constructions in russian. Proceedings of the $27^{\text {th }}$ Annual Penn Linguistics Colloquium. 10 (1), pp. 17-31.

Baio, Jon et al. (2018) Prevalence of autism spectrum disorder among children aged 8 Years. Autism and developmental disabilities monitoring network. MMWR Surveillance Summaries. doi: 10.15585/mmwr.ss6706a1

Barreira, Gabriella (2011) Estudo da morfologia e da sintaxe da linguagem de indivíduos autistas de alto desempenho. Dissertação de Pós-graduação, Instituto Fernandes Figueira.

Bever, Thomas G. (1970) The cognitive basis for linguistic structures. In John R. Hayes (ed.) Cognition and the development of language, 279-362. New York: John Wiley and Sons, Inc.

Bishop, Dorothy (2017) Why is it so hard to reach agreement on terminology? The case of Developmental Language Disorder (DLD). International Journal of Language and Communication Disorders 52.6, pp. 671-680.

Borer Hagit, Wexler Kenneth (1987) The Maturation of Syntax. In: Roeper T., Williams E. (eds) Parameter Setting. Studies in Theoretical Psycholinguistics, 4. Springer, Dordrecht

Chomsky, Noam (1981) Lectures on Government and Binding. Dordrecht: Foris.

Chomsky, Noam (1995) The Minimalist Program. The MIT Press.

Collins, Chris (2005) - A smuggling approach to the passive in english. Syntax. 8(2), pp. 81-120. doi: 10.1111/j.1467-9612.2005.00076.x

Corrêa, Letícia Maria Sicuro, Augusto, Marina R.A. and Lima Júnior, João Claudio (2017) Passivas. In Freitas, Maria João Freitas; Santos, Ana Lúcia (eds.), Aquisição de língua materna e não materna: Questões gerais e dados do português. Pp: 201-224. Berlin: Language Science Press. DOI:10.5281/zenodo.889433

Costa, João; Friedmann, Naama. - Children acquire unaccusatives and A-movement very early. In Everaert, M.; Marelj, M.; Siloni; T. (Eds.) - The theta system: Argument structure at the interface. Oxford: [s.n.]. pp. 354378. 
Duarte, Inês; Oliveira, Fátima (2010) Particípios Resultativos. Textos Seleccionados, XXV Encontro Nacional da Associação Portuguesa de Linguística, Porto: APL, pp. 397-408

Durrleman, Stephanie et al (2015) - Complex syntax in autism spectrum disorders: A study of relative clauses. International Journal of Language and Communication Disorders. 50(2), pp. 260-267. doi: 10.1111/14606984.12130.

Durrleman, Stephanie; Delage, Hélène; Prèvost, Philippe; Tuller, Laurice; (2017) The comprehension of passives in Autism Spectrum Disorder. Glossa: a journal of general linguistics. 2 (1) doi: 10.5334/gjgl.205

Eigsti, Inge-Marie, Bennetto, Loisa (2009) Grammaticality judgments in autism: Deviance or delay. Journal of Child Language. ISSN 0305-0009. 36 (5), pp. 999-1021. doi: 10.1017/s0305000909009362.

Eigsti, Inge-Marie, Bennetto, Loisa, Dadlani, Mamta B. (2007) Beyond pragmatics: Morphosyntactic development in autism. Journal of Autism and Developmental Disorders. 37 (6), pp. 1007-1023. doi: 10.1007/s10803-006-0239-2.

Estrela, Antónia. (2013) A Aquisição da Estrutura Passiva em Português Europeu. Tese de Doutoramento em Linguística. Universidade Nova de Lisboa

Fombonne, Eric (2009) Epidemiology of pervasive developmental disorders. Pediatric Research. 65 (6), pp. 591-598. doi: 10.1203/PDR.0b013e31819e7203

Fox, Danny, Grodzinsky (1998) Children's passive: a view from the by-phrase. Linguistic Inquiry. 29 (2), pp. 311-322

Gavarró, Anna; Heshmati, Yalda (2014) An investigation on the comprehension of Persian passives in typical development and autism. Catalan Journal of Linguistics. 13, pp. 79-98. doi: 10.5565/rev/catjl.151.

Goodwin, Anthony; Fein, Deborah; Naigles, Letitia R. (2012) Comprehension of wh-questions precedes their production in typical development and autism spectrum disorders. Autism Research. 5(2), pp. 109-123. doi: 10.1002/aur.1220.

Hirsch, Christopher; Wexler, Ken (2006) Children's passives and their resulting interpretation. In The proceedings of the inaugural conference on Generative Approaches to Language Acquisition - North America. Editado por: Deen, K.; Nomura, J.; Schulz, B.; Schwartz, B.; University of Connecticut Occasional Papers in Linguistics. 4, pp. 125-136

Hyams, Nina, Snyder, Willian (2005) Young children never smuggle: Reflexive clitics and the Universal Freezing Hypothesis. Handout presented at the 30th Annual Boston University Conference on Language Development.

Kjelgaard, Margaret M.; Tager-Flusberg, Helen (2001) An investigation of language impairment in autism: Implications for genetic subgroups. Language and Cognitive Processes. 16(2-3), pp. 287-308. doi: 10.1080/01690960042000058.

Klin, Ami (2006) Autismo e síndrome de Asperger: uma visão geral. Rev Bras Psiquiatr. 28, pp. 3-11.

Martins, Alexandrina; Santos, Ana Lúcia; Duarte, Inês (2017) Syntactic complexity in children with Autism Spectrum Disorder and Specific Language Impairment. In Language Processing and Disorders (L. Escobar and V. Torrens ans T. Parodi). Newcastle: Cambridge Scholars Publishing, pp. 291-313.

Martins, Alexandrina, Santos, Ana Lúcia, \& Duarte, Inês (2018) Comprehension os relative clauses vs control strutures in SLI and ASD children. In Proceedings of the 42nd annual Boston University Conference on Language Development (Anne B. Bertolini and Maxwell J. Kaplan) Sommerville, MA: Cascadilla Press, pp. 493-506.

Mateus, Maria Helena Mira; Brito, Ana Maria, Duarte. Inês, Faria, Isabel Hub; Frota, Sónia, Matos G., Oliveira Fátima, Vigário Marina, Villalva, Alina. (2003) Gramática da Língua Portuguesa. Edição revista e aumentada. Lisboa: Editorial Caminho. 
Moita, Mara (no prelo) A aquisição de dependências sintáticas com movimento em crianças surdas com implante colcear: um défice de movimento? Tese de Doutoramento em Linguística. Universidade Nova de Lisboa.

Oliveira, Guiomar et al. (2007) Epidemiology of autism spectrum disorder in Portugal: Prevalence, clinical characterization, and medical conditions. Developmental Medicine and Child Neurology. 49 (10), pp. 726733. doi: 10.1111/j.1469-8749.2007.00726.x.

Paul, Rhea; Fischer, Mary L.; Cohen, Donald, J. (1988) Brief report: sentence comprehension in children with autism and specific language disorders. Journal of Autism and Developmental Disorders. 18. pp. 669-679.

Perovic, Alexandra, Modyanova, Nadya; Wexler, Ken (2007) Knowledge of c-command and A-movement in children and adolescents with autism and with Asperger syndrome. Paper presented at the Generative Approaches to Language Acquisition. Barcelona.

Rubin, Maraci Coelho De Barros Pereira (2001) Compreensão Da Passiva Das Crianças Típicas. In Anais do $6^{\circ}$ Encontro do Celsul Círculo de Estudos Lingüísticos do Sul.

Schroeder, Kristen (2013) What Was Done to Whom? Dissertação de Mestrado, Universitat Autònoma de Barcelona.

Tager-Flusberg, Helen (1981) On the nature of linguistic functioning in early infantile autism. Journal of Autism and Developmental Disorders. 11(1). pp. 45-56.

Tager-Flusberg, Helen; Joseph, Robert M. (2003) Identifying neurocognitive phenotypes in autism. Philosophical Transactions of the Royal Society B. 358 (1430), pp. 303-314. doi: 10.1098/rstb.2002.1198.

Tek, Saime; Mesite, Laura; Fein, Deborah; Naigles, Letitia (2014) Longitudinal analyses of expressive language development reveal two distinct language profiles among young children with autism spectrum disorders. Journal of Autism and Developmental Disorders. 44 (1), pp. 75-89. doi: 10.1007/s10803-013-1853-4.

Terzi, Arhonto, Marinis, Theodoros; Kotsopoulou, Angeliki; Francis, Konstantinus (2014) Grammatical Abilities of Greek-Speaking Children with Autism. Language Acquisition. 21(1), pp. 4-44. doi: 10.1080/10489223.2013.855216.

US EPA. 2019. ACE: Health - Neurodevelopmental Disorders | US EPA. [online] Disponível em: $<$ https://www.epa.gov/americaschildrenenvironment/ace-health-neurodevelopmental-disorders $>$ [Accessed 8 October 2020].

Volpato, Francesca; Verin, Laura; Cardinaletti, Anna (2016) The comprehension and production of verbal passives by Italian preschool-age children. Applied Psycholinguistics. 37, pp. 901-931.

Wexler, Kenneth (2004) Theory of phasal development: Perfection in child grammar. MIT. Working Papers in Linguistics. 48, pp. 159-209.

World Health Organisation (2004) ICD-10: international statistical clasification od diseases and related health problems: tenth revision, $2^{\text {nd }}$ ed. World Health Organization.

Whyte, Elisabeth M.; Nelson, Keit E.; Scherf, K. Suzanne (2014) Idiom, syntax, and advanced theory of mind abilities in children with autism spectrum disorders. Journal of Speech, Language, and Hearing Research. 57(1), pp.120-130. doi: 10.1044/1092-4388(2013/12-0308).

Wing, Lorna; Potter, David (2002) The epidemiology of autistic spectrum disorders: Is the prevalence rising? Mental Retardation and Developmental Disabilities Research Reviews. 8(3), pp. 151-161. doi: $10.1002 /$ mrdd.10029.

Wittke, Kacie et al. (2017) Grammatical language impairment in autism spectrum disorder: Exploring language phenotypes beyond standardized testing. Frontiers in Psychology. 8, pp. 1-12. doi: 10.3389/fpsyg.2017.00532. 